\title{
Orthorexia Nervosa: over concern or obsession about healthy food?
}

\author{
Caterina Novara $^{1}$ (1) Susanna Pardini ${ }^{1} \cdot$ Eleonora Maggio ${ }^{1} \cdot$ Sofia Mattioli ${ }^{1} \cdot$ Sara Piasentin $^{1}$
}

Received: 10 August 2020 / Accepted: 13 January 2021 / Published online: 10 February 2021

(c) The Author(s) 2021

\begin{abstract}
Purpose Orthorexia Nervosa is characterized by specific behaviors frequently related to other psychopathological conditions, such as Obsessive-Compulsive Disorder (OCD) and Eating Disorders (EDs). Whereas ON can mainly be described as an excessive concern regarding healthy food, the study's principal aim was to investigate if ON could be considered a condition related and differentiated from worry, other than OCD, EDs, perfectionism, anxiety, and depression.

Method To achieve these aims, 302 individuals from the general population were enrolled and were divided into two groups named "High EHQ" and "Low EHQ", based on their Eating Habits Questionnaire's score (EHQ-21).

Results Correlations of ON with EDs and non-adaptive perfectionism constructs emerged independently from ObsessiveCompulsive (OC) symptoms, and the same pattern was observed when comparing the High and the Low EHQ groups. The two groups also differ in the worry anxiety and depression constructs and are not affected by OC symptoms removal.

Discussion Our results confirm a relationship between ON with the typical ED, perfectionistic, anxious, and depressive symptomatology, mainly when the OC features are controlled; moreover, worry constructs could be considered characteristic of the ON phenomenology. This study does not entirely exclude the relationship with obsessive and compulsive characteristics, which could be associated with or serve as a mediator of the orthorexic behavior. Future research could explore the potential mediating or collateral role of OC symptoms.
\end{abstract}

Level of evidence Level III, evidence obtained from well-designed cohort or case-control analytic studies.

Keywords Orthorexia Nervosa $\cdot$ Worry $\cdot$ Eating features $\cdot$ Obsessive-compulsive features $\cdot$ Dieting

\section{Introduction}

In 1997, Bratman coined the term Orthorexia Nervosa $(\mathrm{ON})$, referring to a psychological condition characterized by excessive concern about eating healthy food with specific nutritional properties initially chosen to eat healthily, prevent and manage diseases including overweight. Healthy eating habits could also be like a pathological obsession. In this way, time spent to prepare and eat a meal becomes excessive [1-3] and could also relate to health problems (e.g., due to nutritional deficits) $[2,4,5]$. However, ON is still not classified as a psychopathological category by the Diagnostic and Statistical Manual of Mental Disorders-5 (DSM-5) [6]. The differences between a healthy eating style and $\mathrm{ON}$ are still unknown. The same goes for the differences between ON,

Caterina Novara

caterina.novara@unipd.it

1 Department of General Psychology, University of Padova, Via Venezia 8, 35131 Padova, Italy
Eating Disorders (EDs), and over concerns about healthy foods; therefore, research in this field is necessary.

To investigate ON and EDs relation and differences, several studies focused on specific demographic characteristics or nutritional choices, obtaining heterogeneous results about age [7-9], education level [10,11], gender [7, 12, 13], and the Body Mass Index (BMI): most studies showed that BMI is unrelated to $\mathrm{ON}[8,14,15]$. A relationship between high BMI and orthorexic tendencies has been highlighted [16, 17]. Otherwise, Arusoğlu et al. [18] found out that BMI could be an orthorexic tendency's predictor only in combination with EDs and Obsessive-Compulsive (OC) symptoms. Globally, discrepant results could be explained using different measuring instruments: most of these studies used the ORTO-15 [19] and its adapted versions. Indeed, these measures have psychometric limitations, such as the absence of a standardized sample and insufficient construct validity [20-22]. Besides, scholars also used different cut-offs to assess orthorexic symptomatology [20,23]. ON's main features have not yet been identified due to conflicting results in 
the literature and ORTO-15 limitations. In the current study, the EHQ-21 [24] was used to assess ON as it exhibits good psychometric characteristics in different cultures [3, 23, 25].

\section{ON and Eating Disorders}

ON shares some features with other recognized EDs, especially with Anorexia Nervosa (AN) and Bulimia Nervosa (BN) $[11,14,26-32]$. ON and AN/BN imply an over concern about food and lifestyle is modified by an excessive influence of diet's rigid adherence [2, 22, 31, 33]. Differently from $\mathrm{ON}, \mathrm{AN} / \mathrm{BN}$ are mainly focused on quantity rather than the quality of food, and body image disturbances and eating restrictions are driven by fears of gaining weight rather than healthy or ethical reasons [33]. In this regard, control could serve as a maintenance factor for strict eating habits. However, while individuals with AN/BN try to hide their behavior, individuals with ON show it off, exhibiting a superior attitude towards others [2], and may suffer from social isolation and somatic problems [2, 22, 33]. In a more recent study, Zickgraf et al. [31] confirmed ON's partial independence from EDs, emphasizing that the EHQ-21 "Problems" subscale can identify disordered eating habits different from not pathological healthy eating and from EDs.

\section{ON, worry and obsessive-compulsive symptoms}

The literature highlighted relationships between OC symptoms and ON. The content of obsessions in ON usually concerns food and its calories, while rituals tend to be associated with research and preparation of those meals considered healthy in both patients with EDs [27] and the general population [15, 34]. Contaminations or harmful thoughts are the most frequent obsessions, whereas checking and washing rituals are the most frequent compulsions in ON [35, 36]. Moreover, ON features were only moderately associated with Obsessive-Compulsive Disorder (OCD) and EDs symptomatology in a sample of patients with Generalized Anxiety Disorder (GAD) and OCD; in this study, significant differences in ON severity between the two groups has not been shown [37]. Furthermore, in samples with EDs, the relationship between ED and OC symptomatology is confirmed [38, 39].

However, food obsessions prevalently are not ego-dystonic in individuals with ON $[1,33]$, and rituals could not intend to reduce anxiety symptoms related to thought, as it happens in OCD [40, 41]. Indeed, the repetitiveness and permanence of food-related thinking might suggest an excessive concern about the food healthiness and its preparation, rather than an obsession followed by a compulsion to neutralize it.
Excessive attention or concern to prevent unhealthy food intake may lead to developing worries about a healthy, strict diet [37].

\section{ON, perfectionism, depression and anxiety features}

Overlaps with the perfectionism dimensions were often found in populations with OC features or EDs [42]; they have also been linked to ON. A positive association between the maladaptive "Perfectionism" scale of the Eating Disorder Inventory-2 (EDI-2) [43] and greater ON tendencies has been shown [14]. Measured by the Multidimensional Perfectionism Scale (MPS) [44], a relation between perfectionism and orthorexic tendencies in a sample of university students emerged [3, 36]; moreover, a relationship between the "Concern Over Mistakes" MPS subscale and ON tendencies has been highlighted [45]. Symptoms of depression and anxiety are often found positively associated with $\mathrm{ON}$ characteristics [23, 46], as well as with OCD and ED symptoms [47, 48], although in the literature related to ON, opposite results came to light [50].

Scientific literature put in evidence a heterogeneous pattern of features implied in the ON conceptualization with an overlap between EDs (especially, AN and $\mathrm{BN}$ ) and strong relationships with OC $[32,49,50]$ and perfectionism features [3, 14, 32, 36, 45]. To date, while relations between these characteristics are quite evident, associations with worry and independence from obsessions and compulsions have been less investigated.

Based on our knowledge, this is the first study investigating the relationship between $\mathrm{ON}$ and worry and its independence from OC symptomatology. Indeed, the study's principal aim was to examine if ON could be considered a disorder independent from OCD and related to worry's content and cognitive processes, controlling the ObsessiveCompulsive Inventory-R effect. Moreover, other aims were to compare ED, perfectionistic anxiety, and depression features considering two groups of individuals with High and Low EHQ scores, respectively. It is expected that removing OC features, relationships between $\mathrm{ON}$ and $\mathrm{ED}$, perfectionism, anxiety, and depression characteristics would become more evident.

\section{Methods}

\section{Participants}

The study group consisted of 302 students recruited from the university student population in Northern Italy, using snowball sampling. The first small group of students provided the 
contacts of other university students who were subsequently contacted for participation in the research. Initially, data were collected from 310 participants, but eight individuals were excluded from the analysis because of exclusion criteria (presence of mental or neurological disease) identified using a demographic schedule.

The final sample was divided into two groups named, respectively, "High EHQ" $(n=43)$ (it included those who scored higher than 50 on the Eating Habits Questionnaire; corresponding to the 90th percentile based on the total sample scores) and "Low EHQ" $(n=259)$ (EHQ total raw score $<50) .48 .8 \%$ of the first group were female $(n=21)$, with a mean age of 20.60 years $(\mathrm{SD}=2.44$; ranging from 18 to 31 years) and a mean of 14.81 (1.23) years of education. This group had a BMI mean of $21.44(\mathrm{SD}=2.02)$. As far as the "Low EHQ" group is concerned, $58.5 \%$ were female, with a mean age of 20.83 ( $S D=3.33$; ranging from 18 to 49 years $)$, a mean of $14.90(\mathrm{SD}=1.58)$ years of education, and a BMI mean of $21.30(\mathrm{SD}=2.56)$. Considering the demographic variables age, BMI, and years of education, no statistically significant differences between the two groups were found (Fisher $F$ ranging from 0.17 to 0.12 ; n.s.), and the same occurred considering the variable gen$\operatorname{der}\left(\chi^{2}=1.39 ;\right.$ n.s. $)$.

Individuals participated voluntarily and gave their written consent before taking part in the study. Participants were fully informed about the research; moreover, the anonymity and the confidentiality of the collected data were guaranteed.

They responded to a series of paper-and-pencil self-report questionnaires in a single session that lasted approximately $50 \mathrm{~min}$. All measures were administered in a counterbalanced order to avoid any order effects.

This study was conducted according to the Declaration of Helsinki and was approved by the Department of General Psychology' Ethical Committee, University of Padova.

\section{Measures}

Demographic schedule: participants were asked to complete a demographic schedule to collect data about gender, age, education levels, and BMI. Any present mental disorder or neurological disease was investigated as exclusion criteria.

The Eating Habits Questionnaire (EHQ-21) [24]; the Italian version by [23], is a 21 -item self-report questionnaire used to assess Orthorexia Nervosa's features on a fourpoint Likert scale ("False, Not at All True", "Slightly True", "Mainly"). The items are grouped into three subscales named "Knowledge" (it refers to knowledge about healthy eating), "Feelings" (it concerns feelings, emotions, and sensations related to following a healthy diet), and "Problems".
The internal consistency $(0.82<$ Cronbach's $\alpha<0.90)$ and test-retest reliability $(0.72<r<0.81)$ are good.

Psychometric properties of the EHQ-21 within the Italian population were confirmed by an exploratory and confirmatory factorial analysis that put in evidence the original EHQ structure. Moreover, a good internal consistency and a one-month test-retest reliability were highlighted ( $r$ ranging from 0.50 to $0.75 ; 0.001<p<0.01$ ) [23].

The current study showed good internal consistency (Total score: Cronbach's $\alpha=0.89$; "Knowledge": Cronbach's $\alpha=0.79$; "Problems": Cronbach's $\alpha=0.86$ ), except for the "Feelings" subscale, where Cronbach's $\alpha$ is acceptable (Cronbach's $\alpha=0.68$ ).

The Eating Disorder Inventory-3 (EDI-3) [51]; the Italian version by [52], is a 91-item self-report questionnaire used to assess both symptoms and psychological-related eating disorders' features. Items are scored on a six-point Likert scale ("Always", "Usually", "Often", "Sometimes", "Rarely", and "Never"). Specifically, the questionnaire is composed of twelve subscales: three measuring eating disorder symptoms ("Drive for Thinness", "Bulimia", and "Body Dissatisfaction"), and nine investigating different general psychological features considered as some of the risk factors for the development of an EDs ("Low Self-Esteem", "Personal Alienation", "Interpersonal Insecurity", "Interpersonal Alienation", "Interoceptive Deficits", "Emotional Dysregulation", "Perfectionism", "Asceticism", and "Maturity Fears"). The EDI-3 also yields the "Risk for Eating Disorders" (EDI-RISK), a score obtained considering the "Drive for Thinness", "Bulimia", and "Body Dissatisfaction" subscales. Its internal consistency $(0.90<$ Cronbach's $\alpha<0.97)$ and test-retest reliability $(r=0.98)$ were excellent in the validation sample represented by EDs patients. Moreover, the Italian version displayed a good internal consistency $(0.72<$ Cronbach's $\alpha<0.94)$.

For the current study, Cronbach's $\alpha$ displayed a good internal consistency. The "Asceticism" subscale was considered acceptable (Cronbach's $\alpha=0.60$ ) [53] after "item 88 " had been excluded.

The Obsessive Compulsive Inventory-Revised (OCI-R) [54]; the Italian version by [55], is an 18-item self-report questionnaire used to assess OCD symptoms on a five-point Likert scale. The questionnaire is composed of six subscales: "Washing", "Ordering", "Hoarding", "Mental Neutralizing", "Obsessing", and "Checking". Regarding the Italian version, the confirmatory factor analysis proved the original six-factors structure and a good test-retest reliability $(0.76<r<0.99)$.

The current study highlighted good internal consistency (Table 1).

The Beck Anxiety Inventory (BAI) [56]; the Italian version by [57], is a 21 item self-report questionnaire used to assess the severity of anxiety symptoms, with a four-point 
Table 1 Correlations and partial correlations between the Eating Habits Questionnaire and the demographic features and questionnaires

\begin{tabular}{|c|c|c|c|c|c|c|c|c|c|}
\hline & EHQ-Total & $\begin{array}{l}\text { EHQ-Total } \\
\text { W/O } \\
\text { OCI-R Total }\end{array}$ & EHQ-P & $\begin{array}{l}\text { EHQ-P. W/O } \\
\text { OCI-R Total }\end{array}$ & EHQ-K & $\begin{array}{l}\text { EHQ-K. W/O } \\
\text { OCI-R Total }\end{array}$ & EHQ-F & $\begin{array}{l}\text { EHQ-F } \\
\text { W/O } \\
\text { OCI-R Total }\end{array}$ & $\begin{array}{l}\text { Cronbach's } \\
\text { Alpha }\end{array}$ \\
\hline Age & $\begin{array}{l}-0.02 \\
\mathrm{~ns}\end{array}$ & & $\begin{array}{l}-0.04 \\
\mathrm{~ns}\end{array}$ & & $\begin{array}{l}-0.03 \\
\mathrm{~ns}\end{array}$ & & $\begin{array}{l}0.04 \\
\text { Ns }\end{array}$ & & \\
\hline BMI & $\begin{array}{l}0.06 \\
\mathrm{~ns}\end{array}$ & & $\begin{array}{l}0.08 \\
\mathrm{~ns}\end{array}$ & & $\begin{array}{l}0.01 \\
\mathrm{~ns}\end{array}$ & & $\begin{array}{l}0.04 \\
\mathrm{~ns}\end{array}$ & & \\
\hline EDI-3 & & & & & & & & & .95 \\
\hline Drive for Thinness & $\begin{array}{l}.35 \\
<0.001\end{array}$ & $\begin{array}{l}.30 \\
<0.001\end{array}$ & $\begin{array}{l}.36 \\
<0.001\end{array}$ & $\begin{array}{l}.29 \\
<0.001\end{array}$ & $\begin{array}{l}.16 \\
<0.01\end{array}$ & $\begin{array}{l}.14 \\
<0.05\end{array}$ & $\begin{array}{l}.31 \\
<0.001\end{array}$ & $\begin{array}{l}.30 \\
<0.001\end{array}$ & .82 \\
\hline Bulimia & $\begin{array}{l}.22 \\
<0.001\end{array}$ & $\begin{array}{l}.20 \\
<0.01\end{array}$ & $\begin{array}{l}.25 \\
<0.001\end{array}$ & $\begin{array}{l}.22 \\
<0.001\end{array}$ & $\begin{array}{l}.06 \\
\mathrm{~ns}\end{array}$ & $\begin{array}{l}.07 \\
\mathrm{~ns}\end{array}$ & $\begin{array}{l}.18 \\
<0.01\end{array}$ & $\begin{array}{l}.19 \\
<0.01\end{array}$ & .82 \\
\hline Body Dissatisfaction & $\begin{array}{l}.11 \\
\mathrm{~ns}\end{array}$ & $\begin{array}{l}.04 \\
\mathrm{~ns}\end{array}$ & $\begin{array}{l}.10 \\
\mathrm{~ns}\end{array}$ & $\begin{array}{l}.02 \\
\mathrm{~ns}\end{array}$ & $\begin{array}{l}.00 \\
\mathrm{~ns}\end{array}$ & $\begin{array}{l}-.04 \\
\mathrm{~ns}\end{array}$ & $\begin{array}{l}.18 \\
<0.01\end{array}$ & $\begin{array}{l}.15 \\
<0.05\end{array}$ & .86 \\
\hline EDI-Risk & $\begin{array}{l}.26 \\
<0.001\end{array}$ & $\begin{array}{l}.19 \\
<0.01\end{array}$ & $\begin{array}{l}0.26 \\
<0.001\end{array}$ & $\begin{array}{l}.18 \\
<0.01\end{array}$ & $\begin{array}{l}0.08 \\
\mathrm{~ns}\end{array}$ & $\begin{array}{l}.05 \\
\mathrm{~ns}\end{array}$ & $\begin{array}{l}0.28 \\
<0.001\end{array}$ & $\begin{array}{l}.24 \\
<0.001\end{array}$ & \\
\hline Low Self-Esteem & $\begin{array}{l}.00 \\
\mathrm{~ns}\end{array}$ & $\begin{array}{l}-.04 \\
\mathrm{~ns}\end{array}$ & $\begin{array}{l}.09 \\
\text { ns }\end{array}$ & $\begin{array}{l}.04 \\
\mathrm{~ns}\end{array}$ & $\begin{array}{l}-.16 \\
<0.01\end{array}$ & $\begin{array}{l}-.17 \\
<0.01\end{array}$ & $\begin{array}{l}.01 \\
\mathrm{~ns}\end{array}$ & $\begin{array}{l}.00 \\
\mathrm{~ns}\end{array}$ & .84 \\
\hline Personal Alienation & $\begin{array}{l}.10 \\
\mathrm{~ns}\end{array}$ & $\begin{array}{l}.02 \\
\mathrm{~ns}\end{array}$ & $\begin{array}{l}.21 \\
<0.001\end{array}$ & $\begin{array}{l}.16 \\
<0.05\end{array}$ & $\begin{array}{l}-.11 \\
<0.05\end{array}$ & $\begin{array}{l}-.12 \\
\mathrm{~ns}\end{array}$ & $\begin{array}{l}.07 \\
\mathrm{~ns}\end{array}$ & $\begin{array}{l}-.02 \\
\mathrm{~ns}\end{array}$ & .72 \\
\hline Interpersonal Insecurity & $\begin{array}{l}.09 \\
\text { ns }\end{array}$ & $\begin{array}{l}.02 \\
\mathrm{~ns}\end{array}$ & $\begin{array}{l}.19 \\
<0.01\end{array}$ & $\begin{array}{l}.12 \\
\mathrm{~ns}\end{array}$ & $\begin{array}{l}-.06 \\
\mathrm{~ns}\end{array}$ & $\begin{array}{l}-.11 \\
\mathrm{~ns}\end{array}$ & $\begin{array}{l}.03 \\
\mathrm{~ns}\end{array}$ & $\begin{array}{l}-.02 \\
\mathrm{~ns}\end{array}$ & .80 \\
\hline Interpersonal Alienation & $\begin{array}{l}.14 \\
<0.05\end{array}$ & $\begin{array}{l}.05 \\
\mathrm{~ns}\end{array}$ & $\begin{array}{l}.23 \\
<0.001\end{array}$ & $\begin{array}{l}.14 \\
<0.05\end{array}$ & $\begin{array}{l}-.01 \\
\mathrm{~ns}\end{array}$ & $\begin{array}{l}-.08 \\
\mathrm{~ns}\end{array}$ & $\begin{array}{l}.04 \\
\mathrm{~ns}\end{array}$ & $\begin{array}{l}-.01 \\
\mathrm{~ns}\end{array}$ & .73 \\
\hline Interoceptive Deficits & $\begin{array}{l}.24 \\
<0.001\end{array}$ & $\begin{array}{l}.20 \\
<0.01\end{array}$ & $\begin{array}{l}.31 \\
<0.001\end{array}$ & $\begin{array}{l}.23 \\
<0.001\end{array}$ & $\begin{array}{l}.04 \\
\mathrm{~ns}\end{array}$ & $\begin{array}{l}.04 \\
\mathrm{~ns}\end{array}$ & $\begin{array}{l}.20 \\
<0.01\end{array}$ & $\begin{array}{l}.20 \\
<0.001\end{array}$ & .82 \\
\hline Emotional Dysregulation & $\begin{array}{l}.16 \\
<0.01\end{array}$ & $\begin{array}{l}.10 \\
\mathrm{~ns}\end{array}$ & $\begin{array}{l}.22 \\
<0.001\end{array}$ & $\begin{array}{l}.15 \\
<0.05\end{array}$ & $\begin{array}{l}.00 \\
\mathrm{~ns}\end{array}$ & $\begin{array}{l}-.02 \\
\mathrm{~ns}\end{array}$ & $\begin{array}{l}.11 \\
\mathrm{~ns}\end{array}$ & $\begin{array}{l}.08 \\
\mathrm{~ns}\end{array}$ & .77 \\
\hline Perfectionism & $\begin{array}{l}.25 \\
<0.001\end{array}$ & $\begin{array}{l}.18 \\
<0.01\end{array}$ & $\begin{array}{l}.21 \\
<0.001\end{array}$ & $\begin{array}{l}.10 \\
\mathrm{~ns}\end{array}$ & $\begin{array}{l}.21 \\
<0.001\end{array}$ & $\begin{array}{l}.19 \\
<0.01\end{array}$ & $\begin{array}{l}.19 \\
<0.01\end{array}$ & $\begin{array}{l}.17 \\
<0.01\end{array}$ & .71 \\
\hline Asceticism & $\begin{array}{l}.36 \\
<0.001\end{array}$ & $\begin{array}{l}.32 \\
<0.001\end{array}$ & $\begin{array}{l}.38 \\
<0.001\end{array}$ & $\begin{array}{l}.30 \\
<0.001\end{array}$ & $\begin{array}{l}.19 \\
<0.01\end{array}$ & $\begin{array}{l}.19 \\
<0.01\end{array}$ & $\begin{array}{l}.27 \\
<0.001\end{array}$ & $\begin{array}{l}.28 \\
<0.001\end{array}$ & .60 \\
\hline Maturity Fears & $\begin{array}{l}.07 \\
\mathrm{~ns}\end{array}$ & $\begin{array}{l}.06 \\
\mathrm{~ns}\end{array}$ & $\begin{array}{l}.16 \\
<0.01\end{array}$ & $\begin{array}{l}.13 \\
<0.05\end{array}$ & $\begin{array}{l}-.09 \\
\mathrm{~ns}\end{array}$ & $\begin{array}{l}-.07 \\
\mathrm{~ns}\end{array}$ & $\begin{array}{l}.04 \\
\mathrm{~ns}\end{array}$ & $\begin{array}{l}.04 \\
\mathrm{~ns}\end{array}$ & .77 \\
\hline \multicolumn{10}{|l|}{ OCI-R } \\
\hline OCI-R Total & $\begin{array}{l}.31 \\
<0.001\end{array}$ & & $\begin{array}{l}.36 \\
<0.001\end{array}$ & & $\begin{array}{l}.12 \\
<0.05\end{array}$ & & $\begin{array}{l}.21 \\
<0.001\end{array}$ & & .91 \\
\hline OCI-R Washing & $\begin{array}{l}.32 \\
<0.001\end{array}$ & & $\begin{array}{l}.35 \\
<0.001\end{array}$ & & $\begin{array}{l}.17 \\
<0.01\end{array}$ & & $\begin{array}{l}.22 \\
<0.001\end{array}$ & & .76 \\
\hline OCI-R Checking & $\begin{array}{l}.27 \\
<0.001\end{array}$ & & $\begin{array}{l}.32 \\
<0.001\end{array}$ & & $\begin{array}{l}.12 \\
<0.05\end{array}$ & & $\begin{array}{l}.17 \\
<0.01\end{array}$ & & .71 \\
\hline OCI-R Ordering & $\begin{array}{l}.24 \\
<0.001\end{array}$ & & $\begin{array}{l}.27 \\
<0.001\end{array}$ & & $\begin{array}{l}.12 \\
<0.05\end{array}$ & & $\begin{array}{l}.17 \\
<0.01\end{array}$ & & .84 \\
\hline OCI-R Obsessing & $\begin{array}{l}.25 \\
<0.001\end{array}$ & & $\begin{array}{l}.27 \\
<0.001\end{array}$ & & $\begin{array}{l}.11 \\
\mathrm{~ns}\end{array}$ & & $\begin{array}{l}.20 \\
<0.01\end{array}$ & & .80 \\
\hline OCI-R Hoarding & $\begin{array}{l}.18 \\
<0.01\end{array}$ & & $\begin{array}{l}.24 \\
<0.001\end{array}$ & & $\begin{array}{l}.03 \\
\mathrm{~ns}\end{array}$ & & $\begin{array}{l}.10 \\
\mathrm{~ns}\end{array}$ & & .73 \\
\hline $\begin{array}{l}\text { OCI-R Mental Neutral- } \\
\text { izing }\end{array}$ & $\begin{array}{l}.13 \\
<0.05\end{array}$ & & $\begin{array}{l}.20 \\
<0.01\end{array}$ & & $\begin{array}{l}-.02 \\
\text { ns }\end{array}$ & & $\begin{array}{l}.08 \\
\mathrm{~ns}\end{array}$ & & .76 \\
\hline \multicolumn{10}{|l|}{ WDQ } \\
\hline WDQ Total & $\begin{array}{l}.20 \\
<0.001\end{array}$ & $\begin{array}{l}.12 \\
\mathrm{~ns}\end{array}$ & $\begin{array}{l}.24 \\
<0.001\end{array}$ & $\begin{array}{l}.13 \\
<0.05\end{array}$ & $\begin{array}{l}-.02 \\
\mathrm{~ns}\end{array}$ & $\begin{array}{l}-.06 \\
\mathrm{~ns}\end{array}$ & $\begin{array}{l}.26 \\
<0.001\end{array}$ & $\begin{array}{l}.22 \\
<0.001\end{array}$ & .94 \\
\hline WDQ Relationship & $\begin{array}{l}.09 \\
\text { ns }\end{array}$ & $\begin{array}{l}.04 \\
\mathrm{~ns}\end{array}$ & $\begin{array}{l}.14 \\
<0.05\end{array}$ & $\begin{array}{l}.06 \\
\mathrm{~ns}\end{array}$ & $\begin{array}{l}-.07 \\
\mathrm{~ns}\end{array}$ & $\begin{array}{l}-.09 \\
\mathrm{~ns}\end{array}$ & $\begin{array}{l}.15 \\
<0.01\end{array}$ & $\begin{array}{l}.13 \\
<0.05\end{array}$ & .82 \\
\hline $\begin{array}{l}\text { WDQ Lack of Confi- } \\
\text { dence }\end{array}$ & $\begin{array}{l}.15 \\
<0.05\end{array}$ & $\begin{array}{l}.08 \\
\mathrm{~ns}\end{array}$ & $\begin{array}{l}.17 \\
<0.01\end{array}$ & $\begin{array}{l}.08 \\
\mathrm{~ns}\end{array}$ & $\begin{array}{l}-.03 \\
\mathrm{~ns}\end{array}$ & $\begin{array}{l}-.06 \\
\mathrm{~ns}\end{array}$ & $\begin{array}{l}.21 \\
<0.001\end{array}$ & $\begin{array}{l}.18 \\
<0.01\end{array}$ & .86 \\
\hline
\end{tabular}


Table 1 (continued)

\begin{tabular}{|c|c|c|c|c|c|c|c|c|c|}
\hline & EHQ-Total & $\begin{array}{l}\text { EHQ-Total } \\
\text { W/O } \\
\text { OCI-R Total }\end{array}$ & EHQ-P & $\begin{array}{l}\text { EHQ-P. W/O } \\
\text { OCI-R Total }\end{array}$ & EHQ-K & $\begin{array}{l}\text { EHQ-K. W/O } \\
\text { OCI-R Total }\end{array}$ & EHQ-F & $\begin{array}{l}\text { EHQ-F } \\
\text { W/O } \\
\text { OCI-R Total }\end{array}$ & $\begin{array}{l}\text { Cronbach's } \\
\text { Alpha }\end{array}$ \\
\hline WDQ Aimless Future & $\begin{array}{l}.16 \\
<0.01\end{array}$ & $\begin{array}{l}.08 \\
\mathrm{~ns}\end{array}$ & $\begin{array}{l}.19 \\
<0.01\end{array}$ & $\begin{array}{l}.08 \\
\mathrm{~ns}\end{array}$ & $\begin{array}{l}-.02 \\
\mathrm{~ns}\end{array}$ & $\begin{array}{l}-.06 \\
\mathrm{~ns}\end{array}$ & $\begin{array}{l}.21 \\
<0.001\end{array}$ & $\begin{array}{l}.18 \\
<0.01\end{array}$ & .77 \\
\hline $\begin{array}{l}\text { WDQ Work Incompe- } \\
\text { tence }\end{array}$ & $\begin{array}{l}.26 \\
<0.001\end{array}$ & $\begin{array}{l}.17 \\
<0.01\end{array}$ & $\begin{array}{l}.29 \\
<0.001\end{array}$ & $\begin{array}{l}.18 \\
<0.01\end{array}$ & $\begin{array}{l}.05 \\
\mathrm{~ns}\end{array}$ & $\begin{array}{l}.02 \\
\mathrm{~ns}\end{array}$ & $\begin{array}{l}.26 \\
<0.001\end{array}$ & $\begin{array}{l}.23 \\
<0.001\end{array}$ & .75 \\
\hline WDQ Financial & $\begin{array}{l}.21 \\
<0.001\end{array}$ & $\begin{array}{l}.11 \\
\mathrm{~ns}\end{array}$ & $\begin{array}{l}.23 \\
<0.001\end{array}$ & $\begin{array}{l}.12 \\
<0.05\end{array}$ & $\begin{array}{l}.02 \\
\mathrm{~ns}\end{array}$ & $\begin{array}{l}-.04 \\
\mathrm{~ns}\end{array}$ & $\begin{array}{l}.25 \\
<0.001\end{array}$ & $\begin{array}{l}.19 \\
<0.01\end{array}$ & .69 \\
\hline PSWQ & $\begin{array}{l}.15 \\
<0.01\end{array}$ & $\begin{array}{l}.08 \\
\mathrm{~ns}\end{array}$ & $\begin{array}{l}.15 \\
<0.01\end{array}$ & $\begin{array}{l}.06 \\
\mathrm{~ns}\end{array}$ & $\begin{array}{l}.00 \\
\mathrm{~ns}\end{array}$ & $\begin{array}{l}-.03 \\
\mathrm{~ns}\end{array}$ & $\begin{array}{l}.22 \\
<.0 .001\end{array}$ & $\begin{array}{l}.20 \\
<0.01\end{array}$ & .92 \\
\hline \multicolumn{10}{|l|}{ MPS } \\
\hline MPS Total & $\begin{array}{l}.37 \\
<0.001\end{array}$ & $\begin{array}{l}.28 \\
<0.001\end{array}$ & $\begin{array}{l}.30 \\
<0.001\end{array}$ & $\begin{array}{l}.18 \\
<0.01\end{array}$ & $\begin{array}{l}.28 \\
<0.001\end{array}$ & $\begin{array}{l}.26 \\
<0.001\end{array}$ & $\begin{array}{l}.33 \\
<0.001\end{array}$ & $\begin{array}{l}.28 \\
<0.001\end{array}$ & .93 \\
\hline MPS Personal Standard & $\begin{array}{l}.34 \\
<0.001\end{array}$ & $\begin{array}{l}.31 \\
<0.001\end{array}$ & $\begin{array}{l}.23 \\
<0.001\end{array}$ & $\begin{array}{l}.18 \\
<0.01\end{array}$ & $\begin{array}{l}.35 \\
<0.001\end{array}$ & $\begin{array}{l}.34 \\
<0.001\end{array}$ & $\begin{array}{l}.30 \\
<0.001\end{array}$ & $\begin{array}{l}.27 \\
<0.001\end{array}$ & .83 \\
\hline $\begin{array}{l}\text { MPS Concern over } \\
\text { Mistakes }\end{array}$ & $\begin{array}{l}.28 \\
<0.001\end{array}$ & $\begin{array}{l}.20 \\
<0.001\end{array}$ & $\begin{array}{l}.25 \\
<0.001\end{array}$ & $\begin{array}{l}.12 \\
<0.05\end{array}$ & $\begin{array}{l}.18 \\
<0.01\end{array}$ & $\begin{array}{l}.17 \\
<0.01\end{array}$ & $\begin{array}{l}.24 \\
<0.001\end{array}$ & $\begin{array}{l}.22 \\
<0.001\end{array}$ & .90 \\
\hline MPS Doubting of Action & $\begin{array}{l}.32 \\
<0.001\end{array}$ & $\begin{array}{l}.27 \\
<0.001\end{array}$ & $\begin{array}{l}.31 \\
<0.001\end{array}$ & $\begin{array}{l}.23 \\
<0.001\end{array}$ & $\begin{array}{l}.16 \\
<0.01\end{array}$ & $\begin{array}{l}.15 \\
<0.05\end{array}$ & $\begin{array}{l}.31 \\
<0.001\end{array}$ & $\begin{array}{l}.29 \\
<0.001\end{array}$ & .77 \\
\hline $\begin{array}{l}\text { MPS Parental Expecta- } \\
\text { tions }\end{array}$ & $\begin{array}{l}.23 \\
<0.001\end{array}$ & $\begin{array}{l}.16 \\
<0.05\end{array}$ & $\begin{array}{l}.21 \\
<0.001\end{array}$ & $\begin{array}{l}.09 \\
\text { ns }\end{array}$ & $\begin{array}{l}.14 \\
<0.05\end{array}$ & $\begin{array}{l}.14 \\
<0.05\end{array}$ & $\begin{array}{l}.22 \\
<0.001\end{array}$ & $\begin{array}{l}.20 \\
<0.01\end{array}$ & .88 \\
\hline MPS Parental Criticism & $\begin{array}{l}.21 \\
<0.001\end{array}$ & $\begin{array}{l}.12 \\
\mathrm{~ns}\end{array}$ & $\begin{array}{l}.24 \\
<0.001\end{array}$ & $\begin{array}{l}.13 \\
<0.05\end{array}$ & $\begin{array}{l}.08 \\
\mathrm{~ns}\end{array}$ & $\begin{array}{l}.05 \\
\mathrm{~ns}\end{array}$ & $\begin{array}{l}.16 \\
<0.01\end{array}$ & $\begin{array}{l}.10 \\
\mathrm{~ns}\end{array}$ & .74 \\
\hline MPS Organization & $\begin{array}{l}.13 \\
<0.05\end{array}$ & $\begin{array}{l}.07 \\
\mathrm{~ns}\end{array}$ & $\begin{array}{l}.07 \\
\mathrm{~ns}\end{array}$ & $\begin{array}{l}.01 \\
\mathrm{~ns}\end{array}$ & $\begin{array}{l}.18 \\
<0.05\end{array}$ & $\begin{array}{l}.15 \\
<0.05\end{array}$ & $\begin{array}{l}.09 \\
\text { ns }\end{array}$ & $\begin{array}{l}.05 \\
\mathrm{~ns}\end{array}$ & .89 \\
\hline BAI & $\begin{array}{l}.13 \\
<0.05\end{array}$ & $\begin{array}{l}.04 \\
\mathrm{~ns}\end{array}$ & $\begin{array}{l}.14 \\
<0.05\end{array}$ & $\begin{array}{l}.01 \\
\mathrm{~ns}\end{array}$ & $\begin{array}{l}.00 \\
\mathrm{~ns}\end{array}$ & $\begin{array}{l}-.04 \\
\mathrm{~ns}\end{array}$ & $\begin{array}{l}.18 \\
<0.05\end{array}$ & $\begin{array}{l}.15 \\
<0.05\end{array}$ & .90 \\
\hline BDI-II & $\begin{array}{l}.10 \\
\mathrm{~ns}\end{array}$ & $\begin{array}{l}.01 \\
\mathrm{~ns}\end{array}$ & $\begin{array}{l}.16 \\
<0.01\end{array}$ & $\begin{array}{l}.04 \\
\text { ns }\end{array}$ & $\begin{array}{l}-.04 \\
\text { ns }\end{array}$ & $\begin{array}{l}-.06 \\
\mathrm{~ns}\end{array}$ & $\begin{array}{l}.07 \\
\text { ns }\end{array}$ & $\begin{array}{l}.02 \\
\text { ns }\end{array}$ & .90 \\
\hline
\end{tabular}

EHQ-21 Eating Habits Questionnaire; EHQ-P Eating Habits Questionnaire-Problems; $E H Q-K$ Eating Habits Questionnaire-Knowledge; $E H Q-F$ Eating Habits Questionnaire-Feelings; EDI-3 Eating Disorder Inventory-3; EDI-Risk Risk for Eating Disorder considering the "Drive for Thinness", "Bulimia" and "Body Dissatisfaction" subscales; OCI-R Obsessive Compulsive Inventory-Revised; WDQ Worry Domains Questionnaire; $P S W Q$ Penn State Worry Questionnaire; MPS Multidimensional Perfectionism Scale; BAI Beck Anxiety Inventory; BDI-II Beck Depression Inventory-II; W/O Controlling for the OCI-R effect

Likert scale. Both the original and the Italian versions showed good internal consistency (Cronbach's $\alpha=0.92$ and 0.89 , respectively) and adequate test-retest reliability. The current study showed good internal consistency (Table 1).

The Beck Depression Inventory-Second Edition (BDIII) [58]; the Italian version by [59], is a 21-item self-report questionnaire used to assess the severity of depression symptoms, and it is rated on a four-point Likert scale. The original and the Italian versions showed an excellent internal consistency (Cronbach's $\alpha=0.93$ and 0.87 , respectively) and test-retest reliability ( $r=0.93$ and 0.76 , respectively). In the current study, the questionnaire proved to have good internal consistency (Table 1).

The Penn State Worry Questionnaire (PSWQ) [60]; the Italian version by [61], is a 16-item self-report questionnaire based on a five-point Likert scale that assesses the dimensions of traits such as generality, excessiveness, and uncontrollability of pathological worry. Its internal consistency was good (original version: Cronbach's $\alpha=0.93$; Italian version $\alpha=0.85$ ). The current study highlighted excellent internal consistency (Table 1).

The Worry Domains Questionnaire (WDQ) [62, 63]; the Italian version by [61], is a 25-item self-report questionnaire used to assess the specific content of worry. It is divided into five subscales: "Relationship", "Lack of Confidence", "Aimless Future", "Work", and "Financial". Its internal consistency was excellent (original version Cronbach's $\alpha=0.92$ and Italian version $0.65<$ Cronbach's $\alpha<0.90$ ).

The current study showed good internal consistency (Table 1).

The Multidimensional Perfectionism Scale (MPS) [44, 64]; the Italian version by [65], is a 35-item self-report questionnaire rated on a five-point Likert scale. It is administered to assess different traits of perfectionism that are operationalized in six subscales: "Concern over Mistakes", "Personal Standards", "Parental Expectations", "Parental Criticism", 
"Doubting of Actions", and "Organization". The original and the Italian internal consistency are good $(0.76<$ Cronbach's $\alpha<0.93$ ). In the current study, all subscales proved to have good internal consistency (Table 1).

\section{Data analysis}

All analyses were carried out using the SPSS Statistics-Version 22. First of all, Cronbach's alpha was investigated for all the administered self-report questionnaires.

Correlation and partial correlation analysis were used to investigate the relationship between the EHQ and other questionnaires. Multivariate ANOVA, and ANCOVA, and a Chi-squared index were used to explore the differences between groups.

\section{Results}

\section{Correlations with age and BMI}

No correlations were found between the EHQ total/subscales' scores and, respectively, age and BMI.

\section{Correlations with ED characteristics}

Positive correlations between the EHQ total score and the EDI-3 subscales ("Drive for Thinness", "Bulimia", "EDIRISK", "Interoceptive Deficits", "Perfectionism", and "Asceticism" subscales) were highlighted $(0.22<r<0.36$; $p<0.001)$. The same pattern of correlations was found for the EHQ "Problems" subscale and the EDI-3 subscales $(0.25<r<0.38 ; p<0.001)$ and less strongly with "Maturity Fears" $(r=0.16 ; p<0.01)$, "Interpersonal Insecurity" $(r=0.19 ; p<0.01)$, "Emotional Dysregulation" $(r=0.22$; $p<0.001)$, "Interpersonal Alienation" $(r=0.23 ; p<0.001)$, and "Personal Alienation" $(r=0.21 ; p<0.001)$. The EHQ "Knowledge" subscale is positively correlated only with the EDI-3 "Perfectionism", "Asceticism", and "Drive for Thinness" subscales $(0.16<r<0.21 ; p<0.01)$ while it is negatively correlated with the "Low Self-Esteem" subscale $(r=-0.16 ; p<0.01)$. Finally, positive correlations between the EHQ "Feelings" subscale and the EDI-3 "Drive for Thinness", "Bulimia", "Body Dissatisfaction", "EDIRISK", "Interoceptive Deficits", "Perfectionism", "Asceticism" subscales were found $(0.18<r<0.31 ; p<0.01)$. All these relations remained significant if the OCI-R effect was controlled except for the "Interpersonal Insecurity" and "Perfectionism" subscales and the EHQ "Problems" subscale.

\section{Correlations with OC symptoms}

Correlations were also put in evidence between the EHQ total and the OCI-R Total and subscales $(0.13<r<0.31 ; p<0.05)$. The EHQ "Problems" subscale shown positive relations with all the OCI-R subscales and total score $(0.20<r<0.36$; $p<0.01)$, the EHQ "Knowledge" with the OCI-R "Total/ Washing/Checking/Ordering" $(0.12<r<0.17 ; p<0.05)$ and the EHQ "Feelings" subscale with the OCI-R "Total/Washing/Checking/Ordering/Obsessing" subscales $(0.17<r<0.22$; $p<0.01$ ).

\section{Correlations with worry}

Positive relations between the EHQ total score and EHQ "Problems" subscale with the WDQ Total and subscales are highlighted $(0.14<r<0.29 ; p<0.05)(0.15<r<0.26$; $p<0.05)$ except for the "Relationship" subscale. The same pattern of correlations regards the EHQ "Problems" subscale $(0.14<r<0.29 ; p<0.05)$, and the majority of the subscales related are affected by the OCI-R elimination. The EHQ "Feelings" subscale correlates with the total and subscales of the WDQ $(0.15<r<0.26 ; p<0.01)$, and none of these were affected by the OCI-R removal, while the EHQ "Knowledge" subscale did not correlate with any worry constructs.

The PSWQ has a weak correlation with the EHQ total and "Problems" subscale $(r=0.15$ for both $p<0.01)$ and is affected by the OCI-R removal, but the EHQ "Feelings" has a stronger relationship with worry's subscale $(r=0.22 ; p<0.001)$ that remains after removal. No relation emerged with the EHQ "Knowledge".

\section{Correlations with perfectionism}

The EHQ Total is positively related with the MPS Total, "Personal Standard", "Concern over Mistakes", "Doubting of Action", and "Parental Expectations" subscales $(0.23<r<0.37 ; p<0.001)$ and remains still significant after removing the effect of the OCI-R. Other subscales have weaker correlations and are much more affected by the OCI-R removal.

\section{Correlations with anxiety and depression}

Weak or not significant correlations emerged with the BAI and the BDI-II except for the EHQ "Feelings" subscale and the BAI $(r=0.18 ; p<0.05)$ that remain significant after the OCI-R removal. 


\section{Differences between the "High-EHQ" and the "Low-EHQ" groups}

Differences in psychological constructs between the two EHQ groups have been highlighted. Specifically, the "High EHQ" individuals obtained, on average, greater scores compared to the other group considering the EDI-3 and its subscales ("Risk", "Drive for Thinness", "Bulimia", "Personal Alienation", "Interpersonal Insecurity", "Interpersonal Alienation", "Interoceptive Deficits", "Emotional Dysregulation", "Asceticism"; $5.45<F<28.03 ; p<0.05)$. These results remained still significant after controlling for the OCI-R effect $(10.95<F<23.58 ; p<0.00)$. The scales of "Body Dissatisfaction", "Low Self-Esteem", "Perfectionism" and "Maturity Fears" became significant only after removing OCI-R $(5.40<F<13.27 ; p<0.01)$.

The "High EHQ" group differs from the "Low EHQ" one in the OCI-R total and its subscales except for the "Checking" and the "Mental Neutralizing" scales $(4.59<F<7.70$; $p<0.03)$. Groups differ in the content of worry measured through the WDQ $(5.77<F<9.89 ; p<0.02$; $24.46<F<36.12 ; p<0.00)$ with the "Relationship" and "Lack of Confidence" subscales, if the OCI-R total is in covariate $(17.07<F<27.24 ; p<0.00)$, and in the process of worry measured through the PSWQ that become significant after the removal of the OCI-R $(F=17.59 ; p<0.00)$.

Groups differ in the MPS total and its subscales "Personal Standard", "Concern over Mistakes", and "Doubting of Action" subscales $(5.05<F<14.69 ; p<0.03$; controlling for the OCI-R: $7.52<F<30.82 ; p<0.00)$; differences regarding "Parental Expectation" and "Parental Criticism" emerged only after controlling for the OCI-R $(10.42<F<13.79$; $p<0.00)$. Finally, as for the "Organization" subscale, no difference between the groups was found.

The BAI and the BDI-II were found to be different between the two groups only after removing the OCI-R $(19.78<F<22.25 ; p<0.00)$ (Table 2).

\section{Discussion}

Overall, the present study aimed to investigate the relationship between the core features of Orthorexia Nervosa and other psychological constructs. Specifically, one of the aims of this study was to investigate ON's relations with both content and cognitive processes of worry controlling for the OCI-R effects to understand if ON could be considered independent from OC symptomatology. Moreover, other aims were to compare OC, ED, perfectionistic, anxiety, and depression features considering two groups of individuals with High and Low EHQ scores, respectively.

Our results confirm a relationship between ON with ED symptomatology [11, 14, 26-32]. Particularly considering the High-EHQ group when the OC features are controlled. The EHQ subscales are related to the risk of having an Eating Disorder. Specifically, with the constructs of "Drive for Thinness" and "Bulimia", "Interoceptive Deficits", "Perfectionism", and "Asceticism", highlighting and confirming the nuclear factors which combined ON and ED [11, 14, 26-32]. Specifically, the EHQ "Problems" subscale is also related to the "Personal Alienation", "Interpersonal Alienation", "Emotional Dysregulation", and "Maturity Fears" scales confirming the relationship with EDs' dysfunctional aspects. It is interesting to note that relations with ED features, despite a reduction, remain when the OCI-R total score is controlled, underlining ON's independence from OCD characteristics. Differences between the High and Low EHQ features showed the same trend: all the subscales related to eating disorder symptomatology result to differentiate.

Moreover, some subscales ("Body Dissatisfaction", "Low Self-Esteem", "Maturity Fears", and "Perfectionism") differentiated the groups but only if the OCI-R is covariate, showing that $\mathrm{OC}$ features could be considered a confounding factor in the relation between ON and EDs.

Weak relations between ON and worry's contents (assessed by the WDQ) and worry processes (assessed by the PSWQ) have been highlighted and, in general, are affected by the OCI-R removal. Instead, comparisons between the High and Low EHQ groups show significant differences for all the WDQ subscales and the PSWQ that become greater if the OCI-R effect is controlled. Moreover, an increase in eta squared has emerged, highlighting that worry's contents and processes could be considered characteristic of the ON phenomenology. Globally, it is important to highlight how the EHQ "Feelings" subscale is the only one not to be affected by the OCI-R effect in all the correlations, both considering the WDQ and the PSWQ that highlight how ON's emotions are connected with contents and processes of worry rather than OC symptomatology.

As in other studies, obsessive symptoms are positively related both with the EHQ "Total" and "Problems" subscale, showing a partial overlap between constructs (particularly for the symptomatology's severity) [33]. Instead, the "Knowledge" and "Feelings" EHQ subscales are less strongly related, and only with the OCI-R "Washing", "Checking" and "Ordering" subscales that are those that are usually found in samples of individuals with EDs [39, 66]. Finally, only the EHQ "Feelings" subscale is related to the OCI-R "Obsessing" scale highlighting how ON emotions are connected to repetitive thought processes. Moreover, the High and Low EHQ groups comparison has been put in evidence significant differences considering the OCI-R total and subscales, except for the "Mental Neutralizing" scale.

This mixed pattern could be explained, considering that $\mathrm{ON}$ and EDs are not different in the type of process involved as they are in the content. Indeed, ON is similar to EDs 
Table 2 Comparisons between the High and the Low EHQ groups

\begin{tabular}{|c|c|c|c|c|c|}
\hline & $\begin{array}{l}\text { High EHQ } \\
N=37\end{array}$ & $\begin{array}{l}\text { Low EHQ } \\
N=225\end{array}$ & $\begin{array}{l}F\left(_{\text {d.f. }} / \text { OCI-R TOTAL }\right. \\
\left.\text { in covariate }{ }_{\text {d.f. }}\right)\end{array}$ & $\begin{array}{l}p \text { value } \\
\text { OCI-R TOTAL in } \\
\text { covariate }\end{array}$ & $\begin{array}{l}\text { Partial Eta squared/ } \\
\text { OCI-R TOTAL in } \\
\text { covariate }\end{array}$ \\
\hline $\begin{array}{l}\text { EDI Risk } \\
\text { Mean (SD) }\end{array}$ & $27.73(20.43)$ & $20.44(17.11)$ & $5.45_{(1)} / 11.11_{(2)}$ & $0.02 / 0.001$ & $0.021 / 0.08$ \\
\hline $\begin{array}{l}\text { Drive for Thinness } \\
\text { Mean (SD) }\end{array}$ & $9.14(7.33)$ & $5.07(6.01)$ & $13.66_{(1)} / 15.10_{(2)}$ & $0.001 / 0.001$ & $0.05 / 0.10$ \\
\hline $\begin{array}{l}\text { Bulimia } \\
\text { Mean (SD) }\end{array}$ & $6.95(6.33)$ & $4.04(4.73)$ & $10.86_{(1)} / 10.95_{(2)}$ & $0.001 / 0.001$ & $0.04 / 0.08$ \\
\hline $\begin{array}{l}\text { Body Dissatisfaction } \\
\text { Mean (SD) }\end{array}$ & $11.65(9.57)$ & $11.34(9.09)$ & $0.04_{(1)} / 5.40_{(2)}$ & $\mathrm{ns} / 0.01$ & $-/ 0.04$ \\
\hline $\begin{array}{l}\text { Low Self-Esteem } \\
\text { Mean (SD) }\end{array}$ & $5.27(4.62)$ & $4.90(4.79)$ & $0.19_{(1)} / 6.90_{(2)}$ & $\mathrm{ns} / 0.001$ & $-/ 0.05$ \\
\hline $\begin{array}{l}\text { Personal Alienation } \\
\text { Mean (SD) }\end{array}$ & $7.08(4.61)$ & $5.06(4.28)$ & $6.95_{(1)} / 17.10_{(2)}$ & $0.01 / 0.001$ & $0.026 / 0.12$ \\
\hline $\begin{array}{l}\text { Interpersonal Insecurity } \\
\text { Mean (SD) }\end{array}$ & $10.70(5.86)$ & $7.44(5.64)$ & $10.51_{(1)} / 12.65_{(2)}$ & $0.001 / 0.001$ & $0.039 / 0.09$ \\
\hline $\begin{array}{l}\text { Interpersonal Alienation } \\
\text { Mean (SD) }\end{array}$ & $9.05(4.99)$ & $6.35(4.52)$ & $11.06_{(1)} / 16.75_{(2)}$ & $0.001 / 0.001$ & $0.04 / 0.12$ \\
\hline $\begin{array}{l}\text { Interoceptive Deficits } \\
\text { Mean (SD) }\end{array}$ & $9.62(6.86)$ & $5.97(5.71)$ & $12.27_{(1)} / 23.58_{(2)}$ & $0.001 / 0.001$ & $0.045 / 0.15$ \\
\hline $\begin{array}{l}\text { Emotional Dysregulation } \\
\text { Mean (SD) }\end{array}$ & $6.73(5.36)$ & $4.15(4.73)$ & $9.99_{(1)} / 14.31_{(2)}$ & $0.002 / 0.001$ & $0.037 / 0.10$ \\
\hline $\begin{array}{l}\text { Perfectionism } \\
\text { Mean (SD) }\end{array}$ & $7.86(3.79)$ & $6.51(4.45)$ & $3.05_{(1)} / 13.27_{(2)}$ & $\mathrm{ns} / 0.001$ & $-/ 0.09$ \\
\hline $\begin{array}{l}\text { Asceticism } \\
\text { Mean (SD) }\end{array}$ & $7.95(4.48)$ & $4.45(3.58)$ & $28.03_{(1)} / 21.46_{(2)}$ & $0.001 / 0.001$ & $0.10 / 0.14$ \\
\hline $\begin{array}{l}\text { Maturity Fears } \\
\text { Mean (SD) }\end{array}$ & $10.68(6.21)$ & $8.98(5.82)$ & $2.65_{(1)} / 7.45_{(2)}$ & $\mathrm{ns} / 0.001$ & $-/ 0.05$ \\
\hline $\begin{array}{l}\text { OCI Total } \\
\text { Mean (SD) }\end{array}$ & $17.30(13.93)$ & 11.63 (11.07) & $7.70_{(1)}$ & 0.006 & 0.029 \\
\hline $\begin{array}{l}\text { OCI Washing } \\
\text { Mean (SD) }\end{array}$ & $2.95(3.33)$ & $1.52(2.14)$ & $11.78_{(1)}$ & 0.001 & 0.043 \\
\hline $\begin{array}{l}\text { OCI Checking } \\
\text { Mean (SD) }\end{array}$ & $2.59(2.70)$ & $1.81(2.28)$ & $3.53_{(1)}$ & ns & 0.013 \\
\hline $\begin{array}{l}\text { OCI Ordering } \\
\text { Mean (SD) }\end{array}$ & $3.59(3.47)$ & $2.39(2.79)$ & $5.50_{(1)}$ & 0.02 & 0.021 \\
\hline $\begin{array}{l}\text { OCI Obsessing } \\
\text { Mean (SD) }\end{array}$ & $3.68(3.28)$ & $2.57(2.85)$ & $4.59_{(1)}$ & 0.03 & 0.017 \\
\hline $\begin{array}{l}\text { OCI Hoarding } \\
\text { Mean (SD) }\end{array}$ & $3.35(3.15)$ & $2.28(2.53)$ & $5.27_{(1)}$ & 0.023 & 0.02 \\
\hline $\begin{array}{l}\text { OCI Mental Neutralizing } \\
\text { Mean (SD) }\end{array}$ & $1.14(1.65)$ & $1.05(1.97)$ & $0.06_{(1)}$ & $\mathrm{ns}$ & 0.000 \\
\hline $\begin{array}{l}\text { WDQ Total } \\
\text { Mean (SD) }\end{array}$ & $42.51(19.01)$ & 34.05 (17.87) & $7.00_{(1)} / 36.12_{(2)}$ & $0.01 / 0.001$ & $0.03 / 0.22$ \\
\hline $\begin{array}{l}\text { WDQ Relationship } \\
\text { MEAN (SD) }\end{array}$ & $7.54(4.17)$ & $6.62(4.72)$ & $1.24_{(1)} / 17.08_{(2)}$ & $\mathrm{ns} / 0.001$ & $0.005 / 0.12$ \\
\hline $\begin{array}{l}\text { WDQ Lack of Confidence } \\
\text { Mean (SD) }\end{array}$ & $8.81(4.77)$ & $7.27(4.62)$ & $3.52_{(1)} / 27.24_{(2)}$ & $\mathrm{ns} / 0.001$ & $0.01 / 0.17$ \\
\hline $\begin{array}{l}\text { WDQ Aimless Future } \\
\text { Mean (SD) }\end{array}$ & $9.08(4.59)$ & $7.28(3.72)$ & $5.77_{(1)} / 26.71_{(2)}$ & $0.02 / 0.001$ & $0.02 / 0.17$ \\
\hline $\begin{array}{l}\text { WDQ Work Incompetence } \\
\text { Mean (SD) }\end{array}$ & $9.35(4.59)$ & $7.20(3.72)$ & $9.89_{(1)} / 24.46_{(2)}$ & $0.002 / 0.001$ & $0.04 / 0.16$ \\
\hline $\begin{array}{l}\text { WDQ Financial } \\
\text { Mean (SD) }\end{array}$ & $7.73(4.75)$ & $5.67(4.04)$ & $7.82_{(1)} / 25.87_{(2)}$ & $0.01 / 0.001$ & $0.03 / 0.17$ \\
\hline
\end{tabular}


Table 2 (continued)

\begin{tabular}{|c|c|c|c|c|c|}
\hline & $\begin{array}{l}\text { High EHQ } \\
N=37\end{array}$ & $\begin{array}{l}\text { Low EHQ } \\
N=225\end{array}$ & $\begin{array}{l}F\left(_{\text {d.f. }} / \text { OCI-R TOTAL }\right. \\
\text { in covariate } \text { (d.f. }_{\text {di }}\end{array}$ & $\begin{array}{l}p \text { value } \\
\text { OCI-R TOTAL in } \\
\text { covariate }\end{array}$ & $\begin{array}{l}\text { Partial Eta squared/ } \\
\text { OCI-R TOTAL in } \\
\text { covariate }\end{array}$ \\
\hline $\begin{array}{l}\text { PSWQ } \\
\text { Mean (SD) }\end{array}$ & $47.86(13.08)$ & $44.48(12.04)$ & $2.45_{(1)} / 17.59_{(2)}$ & $\mathrm{ns} / 0.001$ & $-/ 0.12$ \\
\hline $\begin{array}{l}\text { MPS Total } \\
\text { Mean (SD) }\end{array}$ & $103.46(17.99)$ & $93.42(20.57)$ & $7.83_{(1)} / 23.04_{(2)}$ & $0.006 / 0.001$ & $0.03 / 0.15$ \\
\hline $\begin{array}{l}\text { MPS Personal Standard } \\
\text { Mean (SD) }\end{array}$ & $24.62(5.44)$ & $21.49(5.59)$ & $10.04_{(1)} / 7.52_{(2)}$ & $0.002 / 0.001$ & $0.04 / 0.06$ \\
\hline $\begin{array}{l}\text { MPS Concern over Mistakes } \\
\text { Mean (SD) }\end{array}$ & $25.51(7.51)$ & $22.58(7.33)$ & $5.05_{(1)} / 19.88_{(2)}$ & $0.03 / 0.001$ & $0.02 / 0.13$ \\
\hline $\begin{array}{l}\text { MPS Doubting of Action } \\
\text { Mean (SD) }\end{array}$ & $12.49(3.31)$ & $10.24(3.31)$ & $14.69_{(1)} / 30.82_{(2)}$ & $0.001 / 0.001$ & $0.05 / 0.19$ \\
\hline $\begin{array}{l}\text { MPS Parental Expectations } \\
\text { Mean (SD) }\end{array}$ & $10.92(3.94)$ & $10.40(4.64)$ & $0.41_{(1)} / 10.42_{(2)}$ & $\mathrm{ns} / 0.001$ & $-/ 0.07$ \\
\hline $\begin{array}{l}\text { MPS Parental Criticism } \\
\text { Mean (SD) }\end{array}$ & $8.30(2.92)$ & $7.76(3.17)$ & $0.93_{(1)} / 13.79_{(2)}$ & $\mathrm{ns} / 0.001$ & $-/ 0.10$ \\
\hline $\begin{array}{l}\text { MPS Organization } \\
\text { Mean (SD) }\end{array}$ & $21.62(4.44)$ & $20.95(5.20)$ & $0.55_{(1)} / 1.29_{(2)}$ & $\mathrm{ns} / \mathrm{ns}$ & $-1-$ \\
\hline $\begin{array}{l}\text { BAI } \\
\text { Mean (SD) }\end{array}$ & $12.62(10.26)$ & $11.12(9.00)$ & $0.86_{(1)} / 22.25_{(2)}$ & $\mathrm{ns} / 0.001$ & $-/ 0.15$ \\
\hline $\begin{array}{l}\text { BDI-II } \\
\text { Mean (SD) }\end{array}$ & $9.73(7.21)$ & $8.04(8.06)$ & $1.44_{(1)} / 19.78_{(2)}$ & $\mathrm{ns} / 0.001$ & $-/ 0.13$ \\
\hline
\end{tabular}

EHQ-21 Eating Habits Questionnaire; EHQ-P Eating Habits Questionnaire-Problems; $E H Q-K$ Eating Habits Questionnaire-Knowledge; $E H Q-F$ Eating Habits Questionnaire-Feelings; EDI-3 Eating Disorder Inventory-3; EDI-Risk Risk for Eating Disorder considering the "Drive for Thinness", "Bulimia" and "Body Dissatisfaction" subscales; OCI-R Obsessive Compulsive Inventory-Revised; WDQ Worry Domains Questionnaire; $P S W Q$ Penn State Worry Questionnaire; MPS Multidimensional Perfectionism Scale; BAI Beck Anxiety Inventory; BDI-II Beck Depression Inventory-II; $d . f$. degrees of freedom; $S D$ Standard Deviation

because both focus on food and ego-syntonicity, but they might have different aims concerning healthy food and weight control [22]. ON processes could be similar to the worry processes, but they may be different from OCD functioning: in ON rituality, preparing food is not implemented to reduce distress, as it occurs for OCD compulsions. Our results support the hypothesis according to which there is a relationship between worry's contents, cognitive processes, and ON features. Unfortunately, the WDQ domains did not allow us to investigate worries related to health. Future studies should analyze health and healthy eating concerns, rather than general contents, differentiating them from typical pathological worry components to confirm this relation. Also, this study's results do not entirely exclude the relationship with OC characteristics, which could be associated with or serve as a mediator of the orthorexic behavior. Future investigations could distinguish beliefs that underlie the compulsive behavior from those that underlie the orthorexic behavior.

In line with previous evidence $[3,36,45]$, relationships between orthorexic characteristics and perfectionism traits are shown in the current study. Indeed, the MPS total score shows a positive correlation both with the EHQ total score and the EHQ "Knowledge", "Problems", and "Feelings" subscales. Above all, the non-adaptive perfectionism, characterized by high personal standards, concern over mistakes, and doubting of actions, continuously correlates with the ON features. Differences between High and Low EHQ are highlighted for the same subscales as well. If the obsessive symptomatology is controlled, these relationships remain substantially unchanged, and all perfectionism components come to light, except for the "Organization" MPS subscale. It is noteworthy that perfectionism is the only construct with a moderate relationship with the EHQ "Knowledge" subscale. Perfectionistic strivings could represent cognitive risk components for the subsequent development of orthorexic symptomatology as it happens in EDs [39].

Correlations with the BAI and the BDI-II are small and are all affected by the obsessive component, except for the EHQ "Feelings" subscale showing a significant correlation with the BAI that persists if the OCI-R effect is controlled. Between the two EHQ groups, covariation with the OCI-R highlights a significant difference as far as anxious and depressive components are concerned. The anxious/depressive component is more evident in the High EHQ group and becomes greater after controlling the OC symptoms. On the whole, it can be assumed that the obsessive component represents a mediating or collateral variable about the 
orthorexic symptomatology, and future studies will consider more accurate models of prediction of the orthorexic components to have an idea to better understand of the importance moderation role that each one has in the ON. Future studies should analyze health and healthy eating concerns, rather than general contents, differentiating them from typical pathological worry components (i.e., work, financial, and relationship issues) to confirm these relations. Besides, this study's results do not entirely exclude the relationship with OC characteristics. Future investigations could distinguish beliefs that underlie the compulsive behavior from those that underlie the orthorexic behavior.

Future studies should investigate the relationship between ON and other mental disorders such as EDs, OCD, and GAD. Orthorexia Nervosa could be a prodromal syndrome, a paucisymptomatic form, or an outcome of a treatment. In the future, research on clinical populations of EDs in comparison with the general population may highlight the role of $\mathrm{ON}$ in EDs. Moreover, it would be important to assess beliefs or metacognitions related to $\mathrm{OCD}$ and GAD to understand their role in ON's occurrence and maintenance.

\section{What is already known on this subject?}

Scientific literature put in evidence a heterogeneous pattern of features implied in the ON conceptualization: some studies highlighted an overlap between EDs (especially, AN and $\mathrm{BN}$ ), instead others have found a strong relation with $\mathrm{OC}$ and perfectionism features.

\section{What this study adds?}

Based on our knowledge, this is the first study which investigated relations between $\mathrm{ON}$ and worry characteristics and its independence from OC symptomatology. Our study highlights that the obsessive component could represents a mediating or collateral variable in relation to the orthorexic symptomatology. In addition, a relationship between $\mathrm{ON}$ and Worry has been put in evidence.

Funding Open Access funding provided by Università degli Studi di Padova.

\section{Compliance with ethical standards}

Conflict of interest The authors whose names are listed immediately below certify that they have no affiliations with or involvement in any organization or entity with any financial interest (such as Honoraria, edicational grants, participation in speakers' bureaus, membership, employment, consultancies, stock ownership, or other equity interest, and expert testimony or patent-licensing arrangements), or non-financial interest (such as personal or professional relationships, affiliations, knowledge or. Beliefs) in the subject matter or materials discussed in this manuscript.

Ethical statement All procedures performed in this study were in accordance with the ethical standards of the institutional research committee and with the 1964 Helsinki Declaration and its later amendments.

Informed consent Informed consent was obtained from all individual participants included in the study.

Open Access This article is licensed under a Creative Commons Attribution 4.0 International License, which permits use, sharing, adaptation, distribution and reproduction in any medium or format, as long as you give appropriate credit to the original author(s) and the source, provide a link to the Creative Commons licence, and indicate if changes were made. The images or other third party material in this article are included in the article's Creative Commons licence, unless indicated otherwise in a credit line to the material. If material is not included in the article's Creative Commons licence and your intended use is not permitted by statutory regulation or exceeds the permitted use, you will need to obtain permission directly from the copyright holder. To view a copy of this licence, visit http://creativecommons.org/licenses/by/4.0/.

\section{References}

1. Bratman S (1997) The health food eating disorder. Yoga $\mathbf{J}$ 42-50. http://www.orthorexia.com

2. Bratman S, Knight D (2000) Orthorexia Nervosa: overcoming the obsession with healthful eating. Health food junkies. Broadway Books, New York

3. Oberle CD, Samaghabadi RO, Hughes EM (2017) Orthorexia nervosa: assessment and correlates with gender, BMI, and personality. Appetite 108:303-310. https://doi.org/10.1016/j.appet .2016.10.021

4. Park SW, Kim JY, Go GJ, Jeon ES, Pyo HJ, Kwon YJ (2011) Orthorexia nervosa with hyponatremia, subcutaneous emphysema, pneumomediastinum, pneumothorax, and pancytopenia. Electrolyte Blood Press 9(1):32-37. https://doi.org/10.5049/ EBP.2011.9.1.32

5. Moroze RM, Dunn TM, Holland CJ, Yager J, Weintraub P (2015) Microthinking about micronutrients: a case of transition from obsessions about healthy eating to near-fatal "orthorexia nervosa" and proposed diagnostic criteria. Psychosomatics 56(4):397-403. https://doi.org/10.1016/j.psym.2014.03.003

6. American Psychiatric Association (2013) Diagnostic and statistical manual of mental disorders, 5th edn. American Psychiatric Publishing, Arlington, VA

7. Depa J, Schweizer J, Bekers SK, Hilzendegen C, StroebeleBenschop N (2017) Prevalence and predictors of orthorexia nervosa among German students using the 21-item-DOS. Eat Weight Disord 22(1):193-199. https://doi.org/10.1007/s4051 9-016-0334-0

8. Reynolds R (2018) Is the prevalence of orthorexia nervosa in an Australian university population $6.5 \%$ ? Eat Weight Disord 23(4):453-458. https://doi.org/10.1007/s40519-018-0535-9

9. Dell'Osso L, Abelli M, Carpita B, Massimetti G, Pini S, Rivetti L, Gorrasi F, Tognetti R, Ricca V, Carmassi C (2016) Orthorexia nervosa in a sample of Italian university population. Rivista Di Psichiatria 51(5):190-196. https://doi.org/10.1708/2476.25888 
10. Ramacciotti CE, Perrone P, Coli E, Burgalassi A, Conversano C, Massimetti G, Dell'Osso L (2011) Orthorexia nervosa in the general population: a preliminary screening using a selfadministered questionnaire (ORTO-15). Eat Weight Disord 16(2):127-130. https://doi.org/10.1007/BF03325318

11. Barnes MA, Caltabiano ML (2017) The interrelationship between orthorexia nervosa, perfectionism, body image and attachment style. Eat Weight Disord 22(1):177-184. https:// doi.org/10.1007/s40519-016-0280-x

12. Almeida C, Vieira Borba V, Santos L (2018) Orthorexia nervosa in a sample of Portuguese fitness participants. Eat Weight Disord Stud 23(4):443-451. https://doi.org/10.1007/s40519-018-0517-y

13. Clifford T, Blyth C (2018) A pilot study comparing the prevalence of orthorexia nervosa in regular students and those in University sports teams. Eat Weight Disord 24(3):473-480. https:// doi.org/10.1007/s40519-018-0584-0

14. Parra-Fernández M-L, Rodríguez-Cano T, Onieva-Zafra MD, Perez-Haro MJ, Casero-Alonso V, Fernández-Martinez E, Notario-Pacheco B (2018) Prevalence of orthorexia nervosa in university students and its relationship with psychopathological aspects of eating behaviour disorders. BMC Psychiatry 18(1):1-8. https://doi.org/10.1186/s12888-018-1943-0

15. Oberle CD, Watkins RS, Burkot AJ (2018) Orthorexic eating behaviors related to exercise addiction and internal motivations in a sample of university students. Eat Weight Disord 23(1):6774. https://doi.org/10.1007/s40519-017-0470-1

16. Roncero M, Barrada JR, Perpiñá C (2017) Measuring orthorexia nervosa: psychometric limitations of the ORTO-15. Span J Psychol 20:1-9. https://doi.org/10.1017/sjp.2017.36

17. Luck-Sikorski C, Jung F, Schlosser K, Riedel-Heller SG (2018) Is orthorexic behavior common in the general public? A large representative study in Germany. Eat Weight Disord 24:267-273. https://doi.org/10.1007/s40519-018-0502-5

18. Arusoglu G, Kabakçi E, Köksal G, Merdol T (2008) Orhorexia nervosa and adaptation of ORTO-11 into Turkish. Turkish J Psychiatry 19(3):283-291. PMID: 18791881

19. Donini LM, Marsili D, Graziani MP, Imbriale M, Cannella C (2005) Orthorexia nervosa: validation of a diagnosis questionnaire. Eat Weight Disord 10(2):28-32. https://doi.org/10.1007/ BF03327537

20. Alvarenga MS, Martins MCT, Sato K, Vargas SVA, Philippi ST, Scagliusi FB (2012) Orthorexia nervosa behavior in a sample of Brazilian dietitians assessed by the Portuguese version of ORTO15. Eat Weight Disord 17(1):29-35. https://doi.org/10.1007/ BF03325325

21. Missbach B, Hinterbuchinger B, Dreiseitl V, Zellhofer S, Kurz C, König J (2015) When eating right, is measured wrong! a validation and critical examination of the ORTO-15 questionnaire in German. PLoS ONE 10(8):1-15. https://doi.org/10.1371/journ al.pone. 0135772

22. Dunn TM, Bratman S (2016) On orthorexia nervosa: a review of the literature and proposed diagnostic criteria. Eat Behav 21:1117. https://doi.org/10.1016/j.eatbeh.2015.12.006

23. Novara C, Pardini S, Pastore M, Mulatti C (2017) Ortoressia Nervosa: un'indagine del costrutto e delle caratteristiche psicometriche della versione italiana dell'Eating Habits Questionnaire-21 (EHQ-21). Psicoterapia Cognitiva e Comportamentale 23(3):291-316

24. Gleaves DH, Graham EC, Ambwani S (2013) Measuring "orthorexia": development of the eating habits questionnaire. Int J Educ Psychol Assess 12(2):1-18. https://doi.org/10.1017/CBO9781107 415324.004

25. Yakın E, Raynal P, Chabrol H (2020) Distinguishing orthorexic behaviors from eating disordered and obsessive-compulsive behaviors: a typological study. Eat Weight Disord. https://doi. org/10.1007/s40519-020-01037-9
26. Dellava JE, Hamer RM, Kanodia A, Reyes-Rodriguezet ML, Bulik CN (2011) Diet and physical activity in women recovered from anorexia nervosa: a pilot study. Int J Eat Disord 44:376-382

27. Segura-Garcia C, Ramacciotti C, Rania M, Aloi M, Caroleo M, Bruni A, De Fazio P (2015) The prevalence of orthorexia nervosa among eating disorder patients after treatment. Eat Weight Disord 20(2):161-166

28. Gramaglia C, Brytek-Matera A, Rogoza R, Zeppegno P (2017) Orthorexia and anorexia nervosa: two distinct phenomena? A cross-cultural comparison of orthorexic behaviours in clinical and non-clinical samples. BMC Psychiatry 17(1):75. https:// doi.org/10.1186/s12888-017-1241-2.PMID:28222705;PMCID :PMC5320801

29. Andreas S, Schedler K, Schulz H et al (2018) Evaluation of a German version of a brief diagnosis questionnaire of symptoms of orthorexia nervosa in patients with mental disorders (Ortho10). Eat Weight Disord 23:75-85. https://doi.org/10.1007/s4051 9-017-0473-y

30. Gramaglia et al (2019) Orthorexia nervosa, eating patterns andpersonality traits: a cross culturalcomparison of Italian, Polish and Spanish university students. BMC Psychiatry 19:235. https://doi. org/10.1186/s12888-019-2208-2

31. Zickgraf HF, Ellis JM, Essayli JH (2019) Disentangling orthorexia nervosa from healthy eating and other eating disorder symptoms: relationships with clinical impairment, comorbidity, and self-reported food choices. Appetite 134:40-49. https://doi. org/10.1016/j.appet.2018.12.006

32. Bartel SJ et al (2020) Classification of Orthorexia Nervosa: further evidence for placement within the eating disorders spectrum. Eat Behav 38:101406. https://doi.org/10.1016/j.eatbeh.2020.101406 (Epub 2020 Jun 6 PMID: 32540715)

33. Cena H, Barthels F, Cuzzolaro M, Bratman S, Brytek-Matera A, Dunn T, Varga M, Missbach B, Donini LM (2019) Definition and diagnostic criteria for orthorexia nervosa: a narrative review of the literature. Eat Weight Disord 24(2):209-246. https://doi. org/10.1007/s40519-018-0606-y

34. Segura-García C, Papaianni MC, Caglioti F, Procopio L, Nisticò CG, Bombardiere L, Capranica L (2012) Orthorexia nervosa: a frequent eating disordered behavior in athletes. Eat Weight Disord 17(4):226-233

35. Çiçekoğlu P, Tunçay GY (2018) A comparison of eating attitudes between vegans/vegetarians and nonvegans/nonvegetarians in terms of orthorexia nervosa. Arch Psychiatr Nurs 32(2):200-205. https://doi.org/10.1016/j.apnu.2017.11.002 (Epub 2017 Nov 7 PMID: 29579513)

36. Hayes O, Wu MS, De Nadai AS, Storch EA (2017) Orthorexia nervosa: an examination of the prevalence, correlates, and associated impairment in a university sample. J Cogn Psychother 31(2):124-135

37. Poyraz CA, Tüfekçioğlu EY, Özdemir A, Baş A, Kani AS, Erginöz E, Duran A (2015) Relationship between Orthorexia and Obsessive-Compulsive Symptoms in Patients with Generalised Anxiety Disorder, Panic disorder and Obsessive Compulsive Disorder. Yeni Symp 53(4):22-26. https://doi.org/10.5455/NYS.20156 10232140265205490

38. Altman SE, Shankman SA (2009) What is the association between obsessive-compulsive disorder and eating disorders? Clin Psychol Rev 29(7):638-646. https://doi.org/10.1016/j.cpr.2009.08.001

39. Pollack LO, Forbush KT (2013) Why do eating disorders and obsessive-compulsive disorder co-occur? Eat Behav 14(2):211215. https://doi.org/10.1016/j.eatbeh.2013.01.004

40. Rachman SJ, Hodgson RJ (1980) Obsessions and compulsions. Prentice-Hall, Englewood Cliffs, N.J.

41. Meier M, Kossakowski JJ, Jones PJ, Kay B, Riemann BC, McNally RJ (2019) Obsessive-compulsive symptoms in eating disorders: 
a network investigation. Int J Eat Disord 53(3):362-371. https:// doi.org/10.1002/eat.23196

42. Limburg K, Watson HJ, Hagger MS, Egan SJ (2017) The relationship between perfectionism and psychopathology: a meta-analysis. J Clin Psychol 73(10):1301-1326. https://doi.org/10.1002/ jclp.22435

43. Garner DM (1991) Eating Disorder Inventory-2: professional manual. Psychological Assessment Resources, Odessa, FL

44. Frost RO, Marten P, Lahart C, Rosenblate R (1990) The dimensions of perfectionism. Cogn Ther Res 14(5):449-468

45. Barrada JR, Roncero M (2018) Bidimensional structure of the orthorexia: development and initial validation of a new instrument. An Psicol-Spain 34(2):283-291. https://doi.org/10.6018/ analesps.34.2.299671

46. Strahler J, Hermann A, Walter B, Stark R (2018) Orthorexia nervosa: a behavioral complex or a psychological condition? J Behav Addict 7(4):1143-1156. https://doi.org/10.1556/2006.7.2018.129

47. Goodwin GM (2015) The overlap between anxiety, depression, and obsessive-compulsive disorder. Dialogues Clin Neurosci 17(3):249-260

48. Miniati M, Benvenuti A, Bologna E, Maglio A, Cotugno B, Massimetti G, Dell'Osso L (2018) Mood spectrum comorbidity in patients with anorexia and bulimia nervosa. Eat Weight Disord 23(3):305-311. https://doi.org/10.1007/s40519-016-0333-1

49. Koven NS, Abry AW (2015) The clinical basis of orthorexia nervosa: emerging perspectives. Neuropsychiatr Dis Treat 11:385. https://doi.org/10.2147/NDT.S61665

50. Brytek-Matera A, Fonte ML, Poggiogalle E, Donini LM, Cena H (2017) Orthorexia nervosa: relationship with obsessive-compulsive symptoms, disordered eating patterns and body uneasiness among Italian university students. Eat Weight Disord 22(4):609_ 617. https://doi.org/10.1007/s40519-017-0427-4 (Epub 2017 Aug 24 PMID: 28840493)

51. Garner DM (2004) Eating Disorder Inventory-3 (EDI-3): professional manual. Psychological Assessment Resources, Odessa, FL

52. Giannini M, Pannocchia L, Dalle Grave R, Muratori F, Viglione V (2008) Eating Disorder Inventory-3. Giunti OS, Firenze

53. Hair, JF, Black WC, Babin BJ, Anderson RE (2010) Multivariate Data Analysis. Prentice Hall, Englewood Cliffs, NJ

54. Foa EB, Huppert JD, Leiberg S, Langner R, Kichic R, Hajcak G, Salkovskis PM (2002) The obsessive-compulsive inventory: development and validation of a short version. Psychol Assess 14(4):485. https://doi.org/10.1037/1040-3590.14.4.485

55. Sica C, Ghisi M, Altoè G, Chiri LR, Franceschini S, Coradeschi D et al (2009) The Italian version of obsessive compulsive inventory: its psychometric properties on community and clinical samples. J Anxiety Disord 23:204-211. https://doi.org/10.1016/j.janxd is.2008.07.001

56. Beck AT, Epstein N, Brown G, Steer RA (1988) An inventory for measuring clinical anxiety: psychometric properties. J Consult Clin Psychol 56:893-897

57. Sica C, Coradeschi D, Ghisi M, Sanavio E (2006) Beck Anxiety Inventory-BAI. Giunti OS, Firenze

58. Beck AT, Steer RA, Brown GK (1996) Manual for the Beck Depression Inventory-II. Psychological Corporation, San Antonio, TX

59. Ghisi M, Flebus GB, Montano A, Sanavio E, Sica C (2006) Beck Depression Inventory-II. Giunti OS, Firenze

60. Meyer TJ, Miller ML, Metzger RL, Borkovec TD (1990) Development and validation of the penn state worry questionnaire. Behav Res Ther 28(6):487-495. https://doi.org/10.1016/00057967(90)90135-6

61. Morani S, Pricci D, Sanavio E (1999) Penn State Worry Questionnaire e Worry Domains Questionnaire. Presentazione delle versioni italiane ed analisi della fedeltà. Psicoterapia cognitiva e comportamentale 5(3):13-34

62. Tallis F, Eysenck M, Mathews A (1992) A questionnaire for the measurement of nonpathological worry. Personality Individ Differ 13(2):161-168

63. Tallis F, Davey GC, Bond A (1994) The worry domains questionnaire. In: Davey GCL, Tallis F (eds) Worrying: perspectives on theory, assessment and treatment. Wiley, New York, pp 285-297

64. Strober J (1998) The Frost Multidimensional Perfectionism Scale Revisited: More perfect with four (instead of six) dimensions. Personality Individ Differ 24:481-491. https://doi.org/10.1016/ S0191-8869(97)00207-9

65. Lombardo C (2008) Adattamento italiano della multidimensional perfectionism scale (MPS). Psicoterapia Cognitiva e Comportamentale 14(3):31-46

66. Hasler G, LaSalle-Ricci VH, Ronquillo JG, Crawley SA, Cochran LW, Kazuba D, Murphy DL (2005) Obsessive-compulsive disorder symptom dimensions show specific relationships to psychiatric comorbidity. Psychiatry Res 135(2):121-132. https://doi. org/10.1016/j.psychres.2005.03.003

Publisher's Note Springer Nature remains neutral with regard to jurisdictional claims in published maps and institutional affiliations. 